\title{
Johannes Jungbauer (2017). Entwicklungspsychologie des Kindes- und Jugendalters. Ein Lehrbuch für Studium und Praxis sozialer Berufe
}

Weinheim: Beltz, ISBN 978-3-7799-3798-2, 226 S., 19,95€

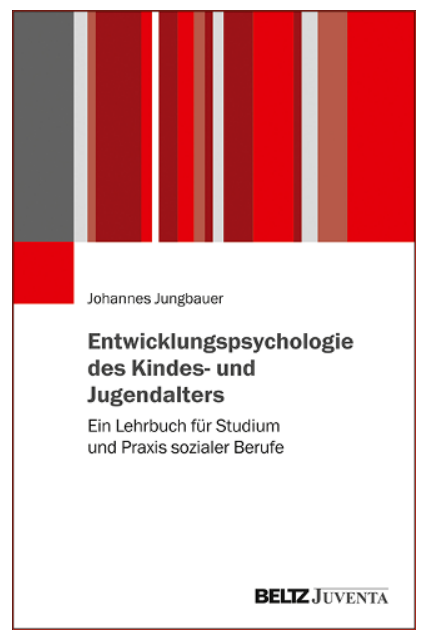

Johannes Jungbauer, Professor für Entwicklungsund Familienpsychologie an der Katholischen Hochschule NordrheinWestfalen in Aachen, hält dort seit 2004 eine Vorlesung zur Entwicklungspsychologie des Kindesund Jugendalters. Seine langjährigen Erfahrungen mit dieser Vorlesung bildeten das Fundament für das vorliegende Lehrbuch. Mit seinem Buch will der Autor „einen kompakten und gut lesbaren Überblick zu zentralen Forschungsfragen, Theorien und Erkenntnissen der Entwicklungspsychologie des Kindes- und Jugendalters" geben (S. 7).

Das Lehrbuch umfasst 13 Kapitel, die zwar inhaltlich immer wieder aufeinander verweisen, aber auch unabhängig voneinander gelesen werden können. Zu Beginn werden wichtige theoretische Grundlagen der Entwicklungspsychologie dargestellt. Bei den folgenden Kapiteln stehen unterschiedliche Entwicklungsbereiche des Kindes- und Jugendalters im Mittelpunkt: Entwicklung von Wahrnehmung und Motorik, Bindung zwischen Eltern und Kind, Lernen als Grundlage von Entwicklung, kognitive Entwicklung, Spracherwerb und Sprachentwicklung, Entwicklung des Selbst in der Kindheit, Entwicklung von moralischem Denken und Verhalten sowie Erziehung und Entwicklung. Den Abschluss bilden drei Kapitel zu spezifischen Entwicklungen und Entwicklungsrisiken im Jugendalter. Neben körperlichen und sexuellen Entwicklungen in der Pubertät wird auf die Identitätsentwicklung im Jugendalter eingegangen und es werden unterschiedliche Erscheinungsformen von jugendlichem Problemverhalten beschrieben.

Das Buch ist sehr gut strukturiert und allgemeinverständlich geschrieben. Dem Autor gelingt es, komplexe entwicklungspsychologische Zusammenhänge gut verständlich darzustellen, ohne allzu sehr zu vereinfachen. Jedes Kapitel beginnt mit einem kurzen Abstract und endet mit Prüfungsfragen, Denkanstößen und weiterer Literatur sowie Internetlinks zur Vertiefung der Themen der Kapitel. Darüber hinaus sind zahlreiche Beispielkästen, Definitionen, Abbildungen und Fotos zu finden. Die besondere Stärke dieses Werkes ist der durchgängige Praxisbezug der besprochenen entwicklungspsychologischen Grundlagen. Es werden immer wieder Beispiele für konkrete Anwendungsmöglichkeiten der Entwicklungspsychologie in der Praxis beschrieben. So werden z. B. im 4. Kapitel nicht nur unterschiedliche Bindungstypen und deren Auswirkungen auf die kindliche Entwicklung dargestellt, sondern auch praktische Anwendungen der Bindungstheorie, wie z. B. außerfamiliäre Tagesbetreuung von Kleinkindern, Beratung bei Trennung und Scheidung, Unterstützung für Pflege- und Adoptionsfamilien sowie Prävention und Elternberatung.

Zusammengefasst bietet dieses Buch eine kompakte, gut verständliche und praxisorientierte Einführung in die Entwicklungspsychologie des Kindes- und Jugendalters. Daher eignet es sich insbesondere für (angehende) Fachkräfte sozialer Berufe, wie z. B. Erzieher_innen, Sozialpädagog_innen oder Lehrer_innen. Aufgrund des Einbezugs aktueller Entwicklungen im Jugendalter, vor allem der gestiegenen zentralen Bedeutung des Internets und der sozialen Medien für den Lebensalltag von Jugendlichen, ist dieses Buch darüber hinaus auch für fortgeschrittene Leser_innen eine sinnvolle Bereicherung. 